\title{
GRADIENTE EDÁFICO DEFINE AS FITOFISIONOMIAS DO PANTANAL DO ABOBRAL, BRASIL
}

\author{
Roberta Franco Pereira de Queiroz ${ }^{1 *}$, Guilherme Resende Corrêa ${ }^{2}$, Frederico dos Santos \\ Gradella $^{3}$ \& Gabriel Palucci Rosa ${ }^{2}$
}

\begin{abstract}
${ }^{1}$ Universidade Federal de Uberlândia, Instituto de Ciências Agrárias, BR 050, km 78, bloco 1CCG, sala 206, CEP 38408369, Uberlândia, MG, Brasil.

${ }^{2}$ Universidade Federal de Uberlândia, Instituto de Geografia, Laboratório de Pedologia e Estudos da Paisagem, Av. João Naves de Ávila, no 2121, Bairro Santa Mônica, Bloco 1H, CEP 38400-902, Uberlândia, MG, Brasil.

${ }^{3}$ Universidade Federal de Mato Grosso do Sul, Campus de Três Lagoas, Av. Ranulpho Marques Leal, n 3484, Distrito Industria, CEP 79620-080, Três Lagoas, MS, Brasil.

E-mails: robertafpqueiroz@gmail.com (*autor correspondente); correasolos@gmail.com; fregradella@yahoo.com.br; gabrielpalucciufu@gmail.com
\end{abstract}

\begin{abstract}
Resumo: O Pantanal é considerado uma das maiores áreas úmidas no interior do continente Sul-Americano e, ao passar por ciclos anuais de inundação, sua extensa área é colonizada por espécies adaptadas aos diversos ambientes, que variam desde terrestres, sazonalmente alagados e aquáticos. Considerando que as variáveis pedológicas, geomorfológicas e o regime hídrico influenciam diretamente no desenvolvimento da comunidade vegetal, este estudo investigou os primeiros $20 \mathrm{~cm}$ do solo como fator de diferenciação de fitofisionomias nas unidades da paisagem do Pantanal do Abobral, estado do Mato Grosso do Sul, Brasil. Foram coletadas amostras simples $(0-20 \mathrm{~cm})$ de solo, com cinco repetições, em cada unidade de paisagem (Geoambientes), realizando-se análises físicas e químicas. Na Cordilheira, ambiente levemente elevado e livre de inundação, há presença de solo naturalmente fértil e básico, que suporta o estabelecimento de floresta semidecídua com sub-bosque. Na planície de inundação, no Corixo, há presença de mata ciliar sobre solo com características redutoras; na Lagoa Intermitente a constante saturação hídrica favorece vegetação tolerante à inundação e macrófitas aquáticas; enquanto o Campo com Cambará, porção sazonalmente alagada, apresentou a menor fertilidade natural, sendo recoberto por gramíneas e processo inicial de colonização por Vochysia divergens (Myrtales, Vochysiaceae). O estudo demonstrou que as descontinuidades da paisagem e nas formações vegetais são correlacionadas às características físicas e químicas dos solos, provando serem um dos principais estratificadores do ambiente, ao lado do nível do lençol freático, sempre próximo à superfície, característica marcante do Pantanal.
\end{abstract}

Palavras-chave: áreas úmidas; estratificação de ambientes; relação solo-vegetação; variáveis pedológicas.

EDAPHIC GRADIENT AND PHYTOPHYSIOGNOMIES OF PANTANAL DO ABOBRAL, BRAZIL. Pantanal is considered one of the largest wetlands inside South America continent. Through the seasonally flood cycles, Pantanal landscape changes in terrestrial, seasonally flooded and aquatic environments, and is colonized by aquatic and terrestrial species. Pedological and geomorphological factors, as well as the water table fluctuation, directly determine plants communities. Thus, this paper investigated the upper soil layer (depth 0-20 cm) as a phytophysiognomies differentiation factor inside Pantanal do Abobral, state of Mato Grosso do Sul, Brazil. Soil samples were collected $(0-20 \mathrm{~cm})$, in replicates of five, in each of four different landscape 
units (here called Geoenvironments) and analyzed for physical and chemical properties. At Cordilheira, slightly higher landscape unit and non-flooded area, occurs natural fertile and basic soil, which supports semi deciduous forest with scrub woodland. At seasonally flooded Geoenvironments, Corixo (periodic river channel) reported riparian forest over soil with reduction characteristics; Lagoa Intermitente (periodic lake) is frequently flooded and colonized by flood tolerant vegetation and macrophytes; and Campo com Cambará, natural grassland periodically waterlogged, had lower fertility and initial colonization by Vochysia divergens (Myrtales, Vochysiaceae). The present study showed that the Pantanal do Abobral discontinuous landscape and vegetation are correlated to superficial edaphic characteristics, proving that soil, is one of the main environmental stratification factors, together with water table, always close to the surface, a striking feature of Pantanal.

Keywords: environmental stratification; pedological variables; soil-vegetation relationship; wetlands.

\section{INTRODUÇÃO}

Considerado uma das maiores áreas úmidas da América do Sul, o Pantanal está sujeito a um ciclo de inundação anual que define as áreas predominantemente aquáticas ou terrestres, distribui sazonalmente as espécies de fauna e flora e permite o intercâmbio de componentes químicos, sedimentos e biota (Por 1995, Ferreira-Junior 2009). Dessa maneira, o alagamento periódico funciona como a força motriz de seus processos ecológicos, além de ser responsável por um enorme controle hidrológico no interior do continente (Cunha \& Junk 2009). Encontra-se sobre uma depressão tectônica recente e circunda-se de planaltos, onde estão as nascentes de seus principais tributários, que drenam para o interior do Pantanal um enorme volume de água e uma grande carga de sedimentos erodidos. Os sedimentos que se depositam e se acumulam sobre a planície pantaneira, juntamente à descarga hídrica, engendram uma paisagem em constate mudança e evolução (Ab'Saber 1988, Assine 2003, Alho 2005).

Devido à sua localização geográfica, o Pantanal se encontra num espaço de transição e contato com os biomas e ecossistemas vizinhos. Por isso, ao contrário dos demais biomas brasileiros, o Pantanal não tem uma única fisionomia vegetal dominante, mas sim elementos característicos dos Cerrados, da Floresta Amazônica, dos Chacos e da Mata Atlântica. Assim, nos alternados ciclos de estiagem e cheia, os diferentes pantanais são colonizados por espécies mais ou menos adaptadas a ambientes aquáticos ou terrestres, nas diferentes épocas do ano (Junk et al. 2006).

Nesta fascinante dinâmica os componentes bióticos e abióticos da paisagem possuem estreita relação, influenciando significativamente sua existência mútua. As variáveis pedológicas, geomorfológicas e o regime hídrico influenciam diretamente no desenvolvimento da comunidade vegetal. Estudos já atestaram a existência de gradientes ambientais, com habitats preferenciais de ocorrência de certas espécies vegetais no Pantanal (Ferreira-Junior 2009, Schaefer et al. 2012, Costa 2013). Isto ocorre devido aos solos e sedimentos constantemente alagados terem seu equilíbrio de elementos e compostos alterados, desencadeando uma série de transformações físicas, químicas e biológicas que influenciam diretamente não apenas formações vegetais, mas a distribuição espacial das espécies (Sousa et al. 2009). Soma-se a isto a grande heterogeneidade de sedimentos que ao longo dos anos formou um mosaico ambiental de classes de solos no Pantanal (Ferreira-Junior 2009).

As unidades da paisagem no Pantanal possuem dinâmica própria de nutrientes, em equilíbrio com a cobertura vegetal e o movimento da água no solo (Cunha 1985). No entanto, devido à diversidade da paisagem, a interpretação dos processos de causa e efeito é sempre complexa e nem sempre aplicável a todos os casos, o que expressa a importância da avaliação particular nas diferentes regiões e ambientes do Pantanal.

A vegetação tem grande controle na distribuição vertical de nutrientes no solo, sendo que suas características vegetacionais, que variam de acordo com a fitofisionomia, são capazes de influenciar o perfil de distribuição desses nutrientes. Ademais, os primeiros $20 \mathrm{~cm}$ do solo são onde se encontram a maior parte dos nutrientes, de tal maneira que é a camada mais explorada pelas raízes finas das plantas (Jobbágy \& Jackson 2001). É também 
a camada em que há maior interação organomineral e onde a ciclagem de nutrientes tem maior expressão, constituindo uma importante camada para investigar a relação solo-vegetação (Novais et al. 2007, Ferreira-Junior 2009). Neste contexto, o trabalho investigou os solos como fator de diferenciação de fitofisionomias nas unidades da paisagem do Pantanal do Abobral, estado do Mato Grosso do Sul, Brasil.

\section{MATERIAL E MÉTODOS}

\section{Área de estudo}

O Pantanal se diferencia em onze sub-regiões, baseando-se em características de inundação, relevo, solo e vegetação (Silva \& Abdon 1998). O Pantanal do Abobral constitui a menor subregião, com $2.833 \mathrm{~km}^{2}$, e corresponde à planície de inundação comum dos rios Abobral, Miranda e Negro (Ravaglia et al. 2010; Figura 1). A região ocupa uma faixa a oeste do estado do Mato Grosso do Sul, inserida nos municípios de Corumbá e Aquidauana. É considerada um corredor de biodiversidade e nela ainda ocorre parte da unidade de conservação Parque Estadual do Rio Negro (Sepúlveda 2016, Andrade 2017).

O Pantanal do Abobral é uma planície mais rebaixada, sendo uma das primeiras a inundar e passa por alagamento generalizado em época de cheia (Cunha et al. 1985, Ravaglia et al. 2010). O clima predominante, segundo a classificação de Köppen, é Aw, clima de Savana, com temperatura média anual de $26^{\circ} \mathrm{C}$. A cheia ocorre no verão, de novembro a março, sendo dezembro e janeiro o período de máxima precipitação. A principal litologia da região é a Formação Pantanal, formada por sedimentos aluviais de granulometria diversa, inconsolidados ou semiconsolidados, cuja espessura sedimentar ultrapassa $500 \mathrm{~m}$ (FerreiraJunior 2009, Couto et al. 2017).

A paisagem do Pantanal do Abobral é dominada por campos limpos e sujos sujeitos
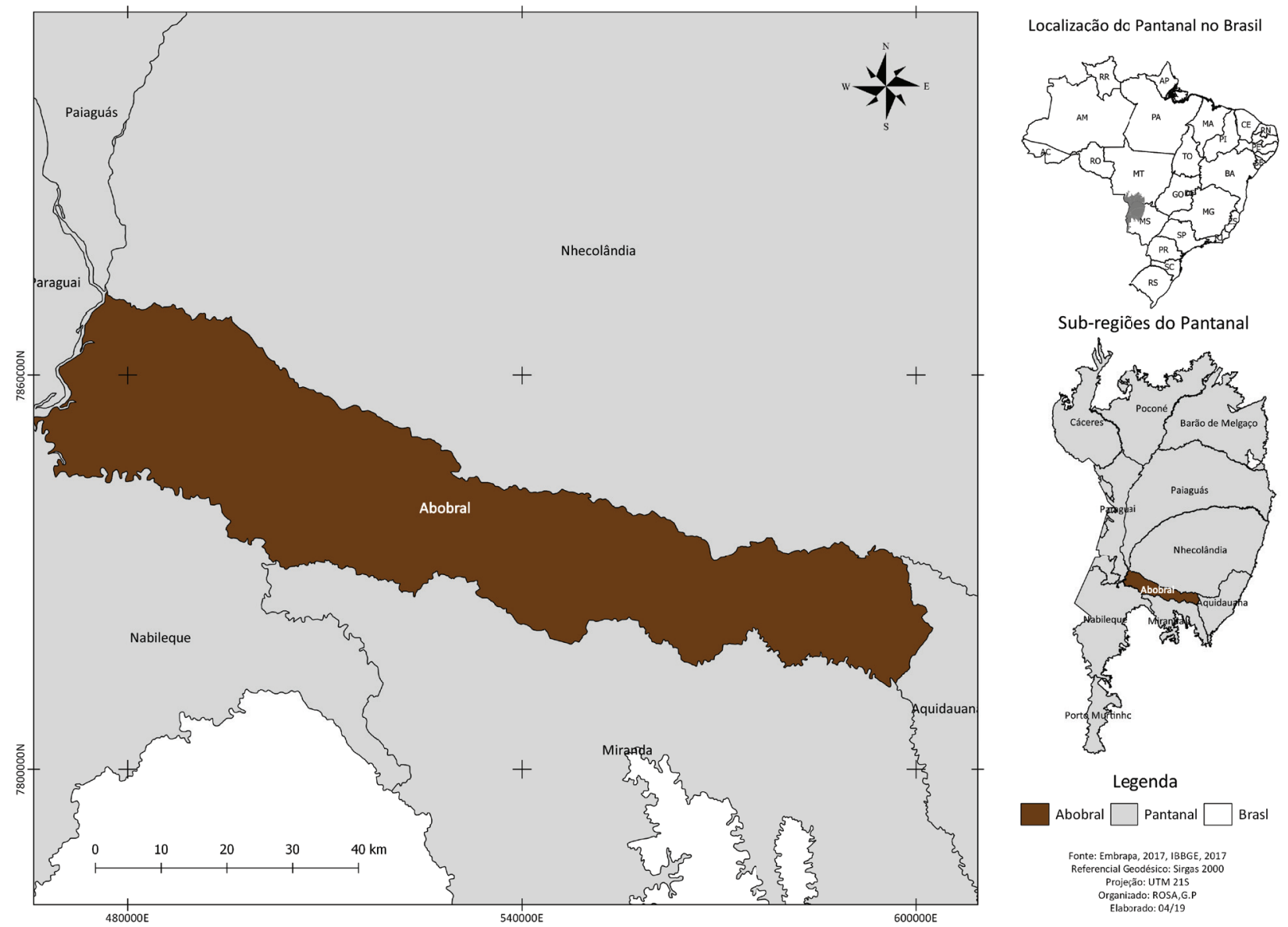

Figura 1. Localização do Pantanal do Abobral, estado do Mato Grosso do Sul, Brasil.

Figure 1. Location of Pantanal do Abobral, state of Mato Grosso do Sul, Brazil. 
a inundação, interrompidos por áreas de mata, chamadas de cordilheiras (Andrade 2017). As unidades da paisagem do Pantanal do Abobral são hierarquizadas em quatro ambientes, chamadas de Geoambientes, segundo suas características de tipo de solo, relevo, material geológico, grau de inundação e fitofisionomia (Queiroz et al. 2017; Tabela 1).

\section{Amostragem do solo}

Para caracterização dos solos, foram coletadas cinco amostras simples da camada superficial (0$20 \mathrm{~cm}$ ) em cada Geoambiente. A coleta dos solos ocorreu em agosto de 2016, momento de estiagem. Uma descrição detalhada dos horizontes dos solos de cada Geoambiente pode ser verificada em Queiroz et al. (2017).

As amostras de solo foram secas ao ar, destorroadas e passadas em peneira de $2 \mathrm{~mm}$ de abertura de malha para obtenção da terra fina seca ao ar (TFSA), que foram submetidas a análises de acordo com os métodos descritos por EMBRAPA (2017). A análise textural foi realizada pelo método da pipeta com utilização de agitação lenta de $50 \mathrm{rpm}$ por $16 \mathrm{~h}$ e determinação do silte por pipetagem. $\mathrm{O}$ $\mathrm{pH}$ foi determinado em água e em solução de $\mathrm{KCl}$ $1 \mathrm{~mol} \mathrm{~L}^{-1}$ em suspensão solo/solução na proporção
1:2,5. Os cátions trocáveis foram extraídos por $\mathrm{KCl}$ $1 \mathrm{~mol} \mathrm{~L}^{-1}$ e quantificados por espectrofotometria de absorção atômica $\left(\mathrm{Ca}^{2+} \mathrm{e} \mathrm{Mg}^{2+}\right)$ e por titulometria com $\mathrm{NaOH}\left(\mathrm{Al}^{3+}\right)$. A acidez potencial $\left(\mathrm{H}^{+}+\mathrm{Al}^{3+}\right)$ foi extraída por acetato de cálcio $0,5 \mathrm{~mol} \mathrm{~L}^{-1} \mathrm{a} \mathrm{pH}$ 7,0 e quantificada por titulometria com $\mathrm{NaOH}$. O $\mathrm{P}$ disponível, $\mathrm{Na}^{+}, \mathrm{K}^{+}$e micronutrientes trocáveis $\left(\mathrm{Cu}^{2+}, \mathrm{Zn}^{2+}, \mathrm{Fe}^{2+}\right.$ e $\left.\mathrm{Mn}^{2+}\right)$ foram extraídos pelo Mehlich-1, sendo P quantificado pelo método do ácido ascórbico, e os demais determinados por espectrofotômetro de plasma induzido. O carbono orgânico (C) foi determinado por Walkley Black sem aquecimento.

As variáveis químicas e físicas dos solos foram submetidas à análise de variância (ANOVA) para verificar a diferença dos atributos pedológicos entre as diferentes unidades da paisagem. Em seguida realizou-se a comparação de médias pelo teste Tukey, quando atenderam às pressuposições para o teste paramétrico, e teste Kruskal-Wallis quando não atenderam tais pressuposições. Ambos os testes utilizaram $5 \%$ de significância, e foram realizados no programa Action (versão 2.9).

Para verificar o efeito dos atributos do solo na separação dos Geoambientes e melhor visualizar as relações de similaridade, foi utilizada a Análise de Componentes Principais (PCA). Antes disso,

Tabela 1. Caracterização dos Geoambientes identificados no Pantanal do Abobral, estado do Mato Grosso do Sul, Brasil. Adaptado de Queiroz et al. (2017).

Table 1. Characterization of Geoenvironments in Pantanal do Abobral, state of Mato Grosso do Sul, Brazil. Adapted from Queiroz et al. (2017).

Geoambiente

Cordilheira com floresta
semidecídua sobre Chernossolo
petrocálcico

Lagoa Intermitente sobre Planossolo eutrófico

Campo com Cambará sobre Planossolo gleissólico
Pedoambiente

Solos muito eutróficos, bem drenados, com acúmulo de carbonatos biogênicos e horizonte petrocálcico

Solo muito mal drenado, com horizonte B plânico, eutrófico

Solo mal drenado, horizonte B plânico, distrófico

\section{Formação vegetal}

Floresta estacional semidecídua com presença de palmeira bacuri e estratos arbustivo e herbáceo esparsos

Campo hidrófilo/higrófilo com arbustos e macrófitas aquáticas

Savana gramíneo-lenhosa com ocorrência de Vochysia divergens de porte arbóreo

Floresta ciliar semidecídua com rara ocorrência de estratos arbustivo e herbáceo
Corixo com mata inundável sobre Solo mal drenado, eutrófico, com Planossolo vertissólico caráter vértico 
as variáveis foram padronizadas e submetidas à análise de correlação de Pearson $\left(\mathrm{r}^{2}>0,70\right)$ para identificar e remover grupos de variáveis altamente correlacionados. Nesse caso, foram desconsideradas da PCA os valores de $\mathrm{pH}_{\mathrm{KCl}}, \mathrm{H}+\mathrm{Al}$ e Zn. Essas análises foram executadas no software $R$ (versão 3.5.1).

\section{RESULTADOS}

A Cordilheira, de maneira geral, foi o Geoambiente que mais se distinguiu dos demais, uma vez que se diferiu estatisticamente em onze dos vinte e cinco atributos pedológicos avaliados (Tabela 2).

A Cordilheira destacou-se como o Geoambiente de maior fertilidade natural, com os maiores valores de fósforo $\left(\mathrm{P}=159,3 \mathrm{mg} / \mathrm{dm}^{3}\right)$, e bases trocáveis, como o cálcio $\left(\mathrm{Ca}^{+2}=8,9 \mathrm{cmol}_{\mathrm{c}} / \mathrm{dm}^{3}\right)$, magnésio $\left(\mathrm{Mg}=1,5 \mathrm{cmol}_{\mathrm{c}} / \mathrm{dm}^{3}\right)$, sódio $\left(\mathrm{Na}=10,5 \mathrm{mg} / \mathrm{dm}^{3}\right)$, e potássio $\left(\mathrm{K}=154,8 \mathrm{mg} / \mathrm{dm}^{3}\right)$, resultando, assim, num $\mathrm{pH}$ do solo elevado $\left(\mathrm{pH}_{\mathrm{H} 2 \mathrm{O}}=8,2\right)$ (Tabela 2). O teor de matéria orgânica na Cordilheira também se destacou como sendo maior, significativamente diferente dos ambientes na planície de inundação (Tabela 2). Com o valor mais elevado de $\mathrm{pH}$ os íons metálicos tenderam a se precipitar e tornarem-se escassos no complexo de troca, se comparados com os demais Geoambientes, como o caso do ferro (Fe $\left.=1,7 \mathrm{mg} / \mathrm{dm}^{3}\right) ;$ cobre $\left(\mathrm{Cu}=0,1 \mathrm{mg} / \mathrm{dm}^{3}\right)$ e alumínio $\left(\mathrm{Al}=0 \mathrm{cmol}_{\mathrm{c}} / \mathrm{dm}^{3}\right)$ (Tabela 2).

Tabela 2. Atributos do solo (profundidade $0-20 \mathrm{~cm}$ ) nos diferentes Geoambientes no Pantanal do Abobral, estado do Mato Grosso do Sul, Brasil. Valores médios $(\mathrm{N}=5)$ seguidos do desvio padrão. Letras na mesma linha indicam diferença estatística significativa entre Geoambientes determinado pelos testes de Tukey ou Kruskal-Wallis (*). SB - Soma de Bases Trocáveis; t - Capacidade de Troca Catiônica Efetiva; T - Capacidade de Troca Catiônica a pH 7,0; V - Índice de Saturação por Bases; $\mathrm{m}$ - Índice de Saturação por Alumínio; ISNa - Índice de Saturação por Sódio; MO - Matéria Orgânica; P-rem - Fósforo Remanescente; AG - Areia Grossa; $\mathrm{AF}$ - Areia Fina.

Table 2. Soil variables (depth $0-20 \mathrm{~cm}$ ) in different Geoenvironments of Pantanal do Abobral, state of Mato Grosso do Sul, Brazil. Values are averaged data $(N=5)$ followed by standard error. Letters in line indicate statistically significant differences between Geoenvironments as determined the Tukey test and the KruskalWallis test (*). SB - Sum of Extractable Bases; $t$ - Effective Cation Exchange Capacity; $T$ - Cation Exchange Capacity at pH 7.0; V-Base Saturation; $m$ - Aluminum Saturation; ISNa - Sodium Saturation; MO - Organic Matter; P-rem - Remaining Phosphorus; AG - Coarse Sand; AF - Fine Sand.

\begin{tabular}{|c|c|c|c|c|c|c|c|c|c|}
\hline \multirow{2}{*}{$\begin{array}{l}\text { Geoambiente } \\
\mathrm{pH}\end{array}$} & \multirow{2}{*}{$\begin{array}{c}\text { Atributos } \\
\mathrm{H} 2 \mathrm{O}\end{array}$} & \multicolumn{2}{|c|}{ Cordilheira } & \multicolumn{2}{|c|}{$\begin{array}{c}\text { Lagoa } \\
\text { Intermitente }\end{array}$} & \multicolumn{2}{|c|}{$\begin{array}{l}\text { Campo com } \\
\text { Cambará }\end{array}$} & \multicolumn{2}{|l|}{ Corixo } \\
\hline & & $8,2 \pm 0,1$ & $\mathrm{a}$ & $5,8 \pm 0,3$ & $\mathrm{~b}$ & $5,4 \pm 0,1$ & $\bar{c}$ & $5,5 \pm 0,1$ & $\mathrm{c}$ \\
\hline $\mathrm{pH}^{*}$ & $\mathrm{KCl}$ & $7,5 \pm 0,1$ & $\mathrm{a}$ & $4,3 \pm 0,1$ & $\mathrm{ab}$ & $4,1 \pm 0,0$ & $\mathrm{~b}$ & $4,2 \pm 0,4$ & $\mathrm{~b}$ \\
\hline $\mathrm{P}$ & $\mathrm{mg} / \mathrm{dm}^{3}$ & $159,3 \pm 68,2$ & $\mathrm{a}$ & $1,2 \pm 0,6$ & bc & $0,5 \pm 0,3$ & $\mathrm{c}$ & $1,8 \pm 0,7$ & $\mathrm{~b}$ \\
\hline K & $\mathrm{mg} / \mathrm{dm}^{3}$ & $154,8 \pm 46,1$ & $\mathrm{a}$ & $46,0 \pm 20,6$ & $\mathrm{~b}$ & $38,6 \pm 11,6$ & $\mathrm{~b}$ & $105,8 \pm 40,4$ & $\mathrm{a}$ \\
\hline $\mathrm{Na}^{+2}$ & $\mathrm{mg} / \mathrm{dm}^{3}$ & $10,5 \pm 2,8$ & a & $5,0 \pm 2,9$ & $\mathrm{~b}$ & $0,0 \pm 0,0$ & c & $2,0 \pm 1,5$ & bc \\
\hline $\mathrm{Ca}^{+2}$ & $\begin{array}{c}\text { cmolc/ } \\
\mathrm{dm}^{3}\end{array}$ & $8,9 \pm 1,0$ & $\mathrm{a}$ & $1,2 \pm 0,3$ & $\mathrm{bc}$ & $0,5 \pm 0,1$ & $\mathrm{~b}$ & $1,9 \pm 0,9$ & $\mathrm{c}$ \\
\hline $\mathrm{Mg}^{+2} *$ & $\begin{array}{l}\text { cmolc/ } \\
\mathrm{dm}^{3}\end{array}$ & $1,5 \pm 0,7$ & a & $0,2 \pm 0,1$ & $\mathrm{bc}$ & $0,1 \pm 0,0$ & $\mathrm{~b}$ & $0,7 \pm 0,3$ & ac \\
\hline $\mathrm{Al}^{+3} *$ & $\begin{array}{l}\text { cmolc/ } \\
\mathrm{dm}^{3}\end{array}$ & $0,0 \pm 0,0$ & $\mathrm{a}$ & $0,0 \pm 0,0$ & $\mathrm{a}$ & $0,5 \pm 0,3$ & $\mathrm{a}$ & $0,4 \pm 0,3$ & $\mathrm{a}$ \\
\hline $\mathrm{H}+\mathrm{Al}$ & $\begin{array}{l}\text { cmolc/ } \\
\mathrm{dm}^{3}\end{array}$ & $0,2 \pm 0,2$ & $\mathrm{a}$ & $2,4 \pm 0,5$ & $\mathrm{~b}$ & $2,5 \pm 0,4$ & $\mathrm{~b}$ & $3,9 \pm 1,6$ & $\mathrm{~b}$ \\
\hline SB & $\begin{array}{l}\text { cmolc/ } \\
\mathrm{dm}^{3}\end{array}$ & $10,8 \pm 1,0$ & $\mathrm{a}$ & $1,6 \pm 0,3$ & $\mathrm{~b}$ & $0,7 \pm 0,2$ & $\mathrm{c}$ & $2,9 \pm 1,2$ & $\mathrm{~d}$ \\
\hline $\mathrm{t}$ & $\begin{array}{c}\text { cmolc/ } \\
\mathrm{dm}^{3}\end{array}$ & $10,8 \pm 1,0$ & a & $1,6 \pm 0,3$ & $\mathrm{~b}$ & $1,2 \pm 0,3$ & $\mathrm{~b}$ & $3,3 \pm 1,5$ & $\mathrm{c}$ \\
\hline
\end{tabular}

Tabela 2. Continua na próxima página...

Table 2. Continued on next page... 
Tabela 2. ...Continuação

Table 2....Continued

\begin{tabular}{|c|c|c|c|c|c|c|c|c|c|}
\hline \multirow{2}{*}{$\begin{array}{l}\text { Geoambiente } \\
\mathrm{T}\end{array}$} & \multirow{2}{*}{$\begin{array}{c}\text { Atributos } \\
\text { cmolc/ } \\
\mathrm{dm}^{3}\end{array}$} & \multicolumn{2}{|c|}{ Cordilheira } & \multicolumn{2}{|c|}{$\begin{array}{c}\text { Lagoa } \\
\text { Intermitente }\end{array}$} & \multicolumn{2}{|c|}{$\begin{array}{l}\text { Campo com } \\
\text { Cambará }\end{array}$} & \multicolumn{2}{|l|}{ Corixo } \\
\hline & & $11,0 \pm 0,9$ & $\mathrm{a}$ & $3,9 \pm 0,8$ & $\mathrm{~b}$ & $3,3 \pm 0,5$ & $\mathrm{~b}$ & $6,8 \pm 2,7$ & $\mathrm{c}$ \\
\hline V & $\%$ & $97,8 \pm 1,6$ & $\mathrm{a}$ & $39,9 \pm 2,5$ & $\mathrm{~b}$ & $22,7 \pm 4,6$ & $\mathrm{c}$ & $42,8 \pm 6,1$ & $\mathrm{~b}$ \\
\hline $\mathrm{m}^{*}$ & $\%$ & $0,0 \pm 0,0$ & $\mathrm{a}$ & $0,0 \pm 0,0$ & $\mathrm{a}$ & $35,3 \pm 22,5$ & $\mathrm{~b}$ & $10,3 \pm 5,6$ & $a b$ \\
\hline ISNa & $\%$ & $0,4 \pm 0,1$ & a & $0,5 \pm 0,2$ & a & $0,0 \pm 0,0$ & $\mathrm{~b}$ & $0,1 \pm 0,1$ & $\mathrm{~b}$ \\
\hline MO & $\mathrm{dag} / \mathrm{kg}$ & $3,2 \pm 0,7$ & $\mathrm{a}$ & $1,1 \pm 0,3$ & $\mathrm{~b}$ & $1,1 \pm 0,4$ & $\mathrm{~b}$ & $1,5 \pm 0,5$ & $\mathrm{~b}$ \\
\hline PRem & $\mathrm{mg} / \mathrm{l}$ & $32,8 \pm 4,1$ & $\mathrm{a}$ & $41,3 \pm 2,2$ & $\mathrm{a}$ & $39,8 \pm 2,9$ & $\mathrm{a}$ & $35,3 \pm 9,4$ & $\mathrm{a}$ \\
\hline $\mathrm{Cu}$ & $\mathrm{mg} / \mathrm{dm}^{3}$ & $0,1 \pm 0,1$ & a & $1,5 \pm 0,3$ & $\mathrm{c}$ & $0,7 \pm 0,2$ & $\mathrm{~b}$ & $1,4 \pm 0,3$ & $\mathrm{c}$ \\
\hline $\mathrm{Mn}$ & $\mathrm{mg} / \mathrm{dm}^{3}$ & $37,0 \pm 11,2$ & ac & $25,9 \pm 8,5$ & $\mathrm{ab}$ & $13,2 \pm 1,9$ & $\mathrm{~b}$ & $51,8 \pm 20,1$ & $\mathrm{c}$ \\
\hline $\mathrm{Fe}$ & $\mathrm{mg} / \mathrm{dm}^{3}$ & $1,7 \pm 1,4$ & $\mathrm{a}$ & $183,6 \pm 41,6$ & $\mathrm{~b}$ & $200,7 \pm 46,7$ & bc & $470,3 \pm 200,7$ & $\mathrm{c}$ \\
\hline $\mathrm{Zn}$ & $\mathrm{mg} / \mathrm{dm}^{3}$ & $0,8 \pm 0,5$ & $\mathrm{ab}$ & $0,5 \pm 0,1$ & $\mathrm{a}$ & $0,3 \pm 0,1$ & $\mathrm{a}$ & $1,3 \pm 0,3$ & $\mathrm{~b}$ \\
\hline AG & $\mathrm{kg} / \mathrm{kg}$ & $9,5 \pm 0,0$ & $\mathrm{a}$ & $9,3 \pm 0,0$ & $\mathrm{a}$ & $10,7 \pm 0,0$ & $\mathrm{a}$ & $9,2 \pm 0,0$ & $\mathrm{a}$ \\
\hline $\mathrm{AF}$ & $\mathrm{kg} / \mathrm{kg}$ & $52,3 \pm 0,0$ & a & $65,1 \pm 0,0$ & $\mathrm{~b}$ & $80,9 \pm 0,0$ & $\mathrm{c}$ & $73,4 \pm 0,0$ & d \\
\hline Silte & $\mathrm{kg} / \mathrm{kg}$ & $9,8 \pm 0,0$ & $\mathrm{a}$ & $17,2 \pm 0,1$ & $\mathrm{~b}$ & $2,1 \pm 0,0$ & $\mathrm{c}$ & $5,6 \pm 0,0$ & ac \\
\hline Argila & $\mathrm{kg} / \mathrm{kg}$ & $28,5 \pm 0,1$ & $\mathrm{a}$ & $8,3 \pm 0,0$ & $\mathrm{bc}$ & $6,3 \pm 0,0$ & $\mathrm{~b}$ & $11,8 \pm 0,0$ & $\mathrm{c}$ \\
\hline
\end{tabular}

Em relação à textura, todos os Geoambientes apresentaram elevados teores de areia, com destaque para Areia Fina (AF) que se diferiu estatisticamente em todos os Geoambientes (Tabela 2), com o Campo com Cambará com maior teor. A Lagoa Intermitente possuiu a maior quantidade de silte, diferindo-se dos demais. Já a Cordilheira apresentou maior teor de argila.

O triângulo textural (Figura 2) demonstrou que os Geoambientes sazonalmente inundáveis (i.e., Lagoa Intermitente, Corixo e Campo com Cambará) possuíram menos que $15 \%$ de argila em superfície, e se concentraram próximos aos maiores valores de areia (>60\%). Em contraste, a Cordilheira possuiu, superficialmente, cerca de $30 \%$ de argila (Tabela 2 ; Figura 2).

Os Geoambientes inundáveis, de maneira geral, foram mais ácidos em superfície (Tabela 2). O Campo com Cambará teve os menores teores de fósforo $\left(\mathrm{P}=0,5 \mathrm{mg} / \mathrm{dm}^{3}\right)$, cálcio $(\mathrm{Ca}=0,5 \mathrm{mg} /$ $\left.\mathrm{dm}^{3}\right)$, sódio $\left(\mathrm{Na}=0 \mathrm{mg} / \mathrm{dm}^{3}\right)$ e magnésio $(\mathrm{Mg}=$ $0,1 \mathrm{mg} / \mathrm{dm}^{3}$ ), que refletiram-se no menor valor de saturação por bases $(\mathrm{V}=22,7 \%)$ (Tabela 2$)$, este último diferente de todos os demais Geoambientes.

Em relação à capacidade de troca catiônica (CTC) efetiva (valor t), CTC em pH 7 (valor T) e índice de saturação de bases, os maiores teores foram descritos na Cordilheira, seguido pelo Corixo,

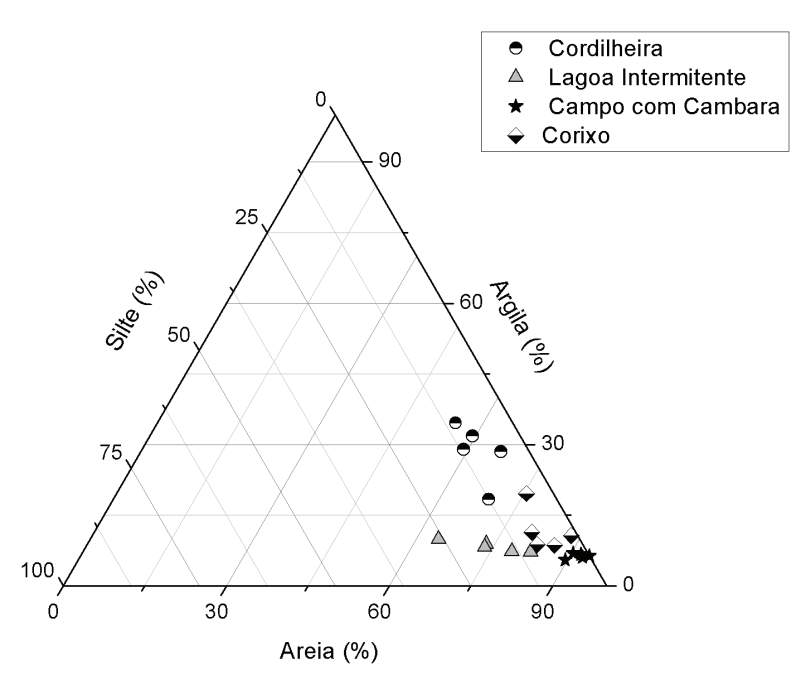

Figura 2. Triângulo textural representativo da camada superficial $(0-20 \mathrm{~cm})$ dos Geoambientes no Pantanal do Abobral, estado do Mato Grosso do Sul, Brasil.

Figure 2. Texture triangle of soil superficial layer (depth $0-20 \mathrm{~cm}$ ) at Pantanal do Abobral Geoenvironments, state of Mato Grosso do Sul, Brazil.

Lagoa Intermitente e Campo com Cambará. Assim, a Cordilheira demonstrou ser o Geoambiente com maior fertilidade natural, e situação oposta é encontrada no Campo com Cambará.

Os íons metálicos se apresentaram mais disponíveis nas unidades da paisagem 
sazonalmente inundáveis. Houve um maior teor de ferro no Corixo $\left(\mathrm{Fe}=470,3 \mathrm{mg} / \mathrm{dm}^{3}\right.$ ), enquanto o menor valor de ferro ocorreu na Cordilheira $(\mathrm{Fe}=$ $1,7 \mathrm{mg} / \mathrm{dm}^{3}$ ), este significativamente diferente dos demais Geoambientes. Em relação ao manganês e zinco, ambos os elementos seguiram o mesmo padrão de arranjo, sendo maiores no Corixo (Mn $=51,8 \mathrm{mg} / \mathrm{dm}^{3}, \mathrm{Zn}=1,3 \mathrm{mg} / \mathrm{dm}^{3}$ ), seguidos da Cordilheira $\left(\mathrm{Mn}=37 \mathrm{mg} / \mathrm{dm}^{3}, \mathrm{Zn}=0,8 \mathrm{mg} / \mathrm{dm}^{3}\right)$, Lagoa Intermitente $\left(\mathrm{Mn}=25,9 \mathrm{mg} / \mathrm{dm}^{3}, \mathrm{Zn}=0,5\right.$ $\mathrm{mg} / \mathrm{dm}^{3}$ ) e Campo com Cambará com os menores valores $\left(\mathrm{Mn}=13,2 \mathrm{mg} / \mathrm{dm}^{3}, \mathrm{Zn}=0,3 \mathrm{mg} / \mathrm{dm}^{3}\right)$ (Tabela 2).

O Geoambiente de Cordilheira apresentou uma fisionomia florestal com abundante ocorrência de palmeira acuri (Scheelea phalerata; Arecales, Arecaceae) (Figura 3a). Houve também presença de angico (Anadenanthera sp.; Fabales, Fabaceae), baru (Dipteryx alata; Fabales, Fabaceae), embaúba (Cecropia pachystachya; Rosales, Urticaceae), amendoeira (Prunus dulcis; Rosales, Rosaceae) e gravatá (Bromelia balansae; Poales, Bromeliaceae). $\mathrm{Na}$ Cordilheira a maior fertilidade natural associouse a uma maior biomassa vegetacional.

A Lagoa Intermitente apresentou vegetação esparsa com algumas macrófitas aquáticas, demonstrando ocorrer seleção da vegetação tolerante à saturação hídrica, ou seja, a deficiência de oxigênio (Figura 3b). A menor fertilidade natural encontrada no Campo com Cambará refletiu-se num parco quadro vegetacional, em que houve dominância de gramíneas e vegetação herbácea pouco palatáveis e colonização inicial por Cambará (Vochysia divergens; Myrtales, Vochysiaceae) (Figura 3c).

O Geoambiente Corixo também apresentou fisionomia florestal, mas diferenciou-se da Cordilheira por ser mais aberta, de menor porte, sujeita a severas inundações periódicas e cerca de oito centímetros de acúmulo de serapilheira (Figura 3d).

A análise de componentes principais (Figura 4) sintetizou a associação dos atributos pedológicos, demonstrando, assim, os fatores de diferenciação em cada Geoambiente. O Eixo 1 explicou cerca de $54 \%$ da variância dos dados, e traduziu as principais diferenças em relação aos atributos nutricionais do solo. Nesse caso, os valores negativos representaram as amostras associadas aos maiores valores nutricionais $(\mathrm{Mg}, \mathrm{Na}, \mathrm{Ca}, \mathrm{K}$,
P), representados unicamente pela Cordilheira. O Campo com Cambará, por sua vez, concentrou-se opostamente aos vetores de nutrientes, reforçando o distinto caráter de fertilidade natural dessas unidades da paisagem (Figura 4).

Os Geoambientes sazonalmente inundáveis ficaram à direita do Eixo 1. A matriz arenosa da Formação Pantanal, juntamente com o processo de podzolização, comum nestes Geoambientes, favoreceram teores elevados de AF nos horizontes superficiais. O maior cumprimento do vetor de areia fina que AG corroborou a maior atuação da AF na distinção dos ambientes. A condição mais ácida da Lagoa Intermitente, Campo com Cambará e Corixo se correlacionou aos vetores de elementos metálicos ( $\mathrm{Al}, \mathrm{Fe}$ e $\mathrm{Cu}$ ), que se tornaram mais disponíveis nestas condições.

\section{DISCUSSÃO}

Os diferentes Geoambientes do Pantanal do Abobral demonstraram estar associados aos atributos edáficos. A disponibilidade de nutrientes e sazonalidade do lençol freático permitem um gradiente fisionômico que varia entre a Cordilheira florestada eutrófica e não alagável e o campo distrófico e alagável.

As Cordilheiras no Pantanal do Abobral representam a única unidade da paisagem predominantemente não inundável (Cunha et al. 1985). Nelas ocorrem Chernossolos, muito ricos em bases trocáveis e influenciados por um intenso depósito de carapaças de caramujos em subsuperfície (Queiroz et al. 2017). Este acúmulo de conchas subsuperficial nos solos da Cordilheira possivelmente contribuiu para os elevados valores de $\mathrm{Ca}^{+2}$ em superfície, componente principal de carapaça de moluscos (formados por aragonita) e $\mathrm{P}$, presente nos tecidos dos gastrópodes e facilmente intemperizável (Corrêa et al. 2011).

O uso do solo na região do Abobral se restringe ao ecoturismo e à pecuária extensiva, esta última muito favorecida pelos campos naturais (Cunha et al. 1985). O gado utiliza frequentemente as Cordilheiras como abrigo na época das cheias, e a presença recorrente de fezes desses animais no local gera um incremento de matéria orgânica nesse Geoambiente. Por ser um pedoambiente predominantemente oxidante, permite uma ciclagem mais rápida de matéria orgânica e 

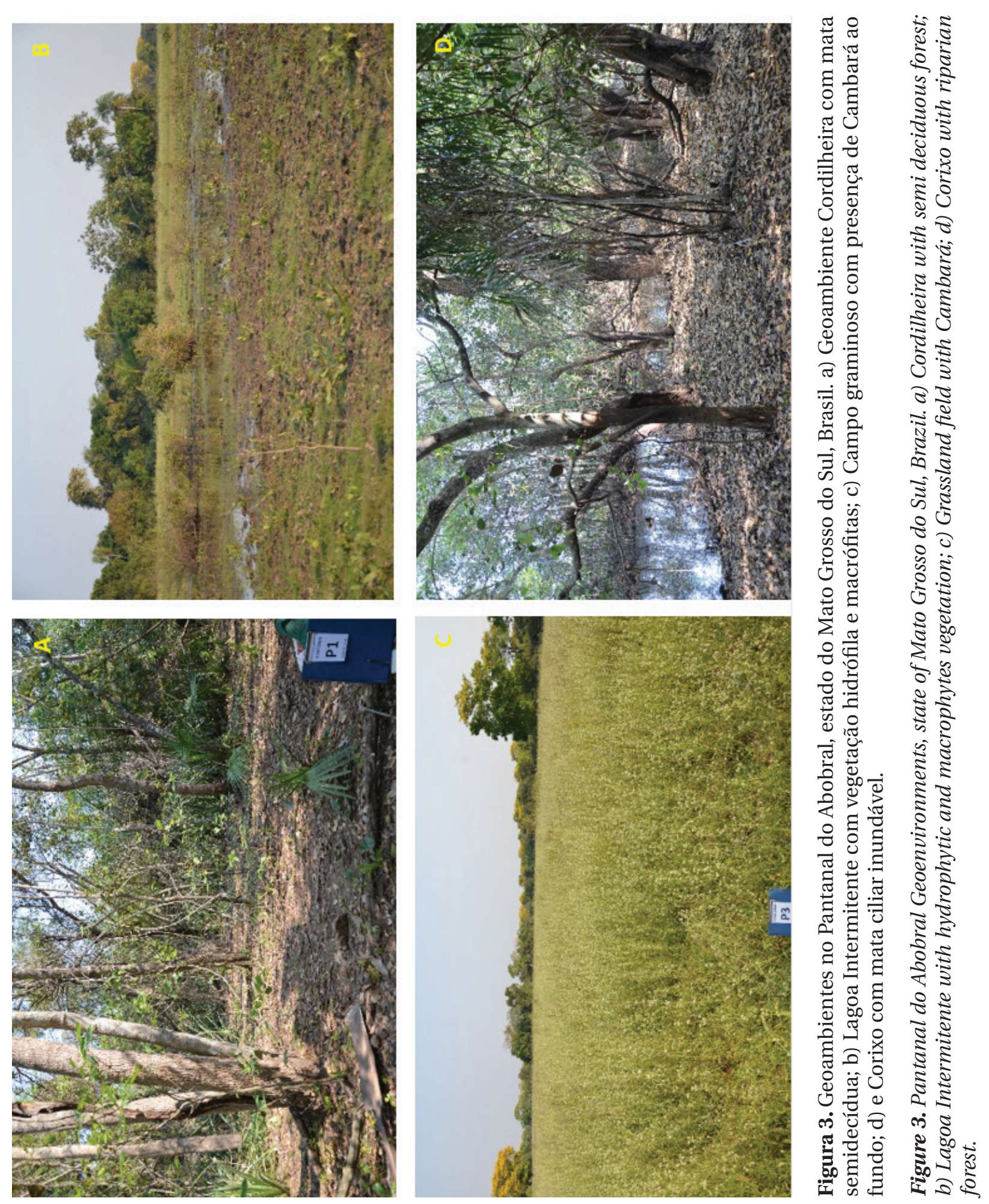


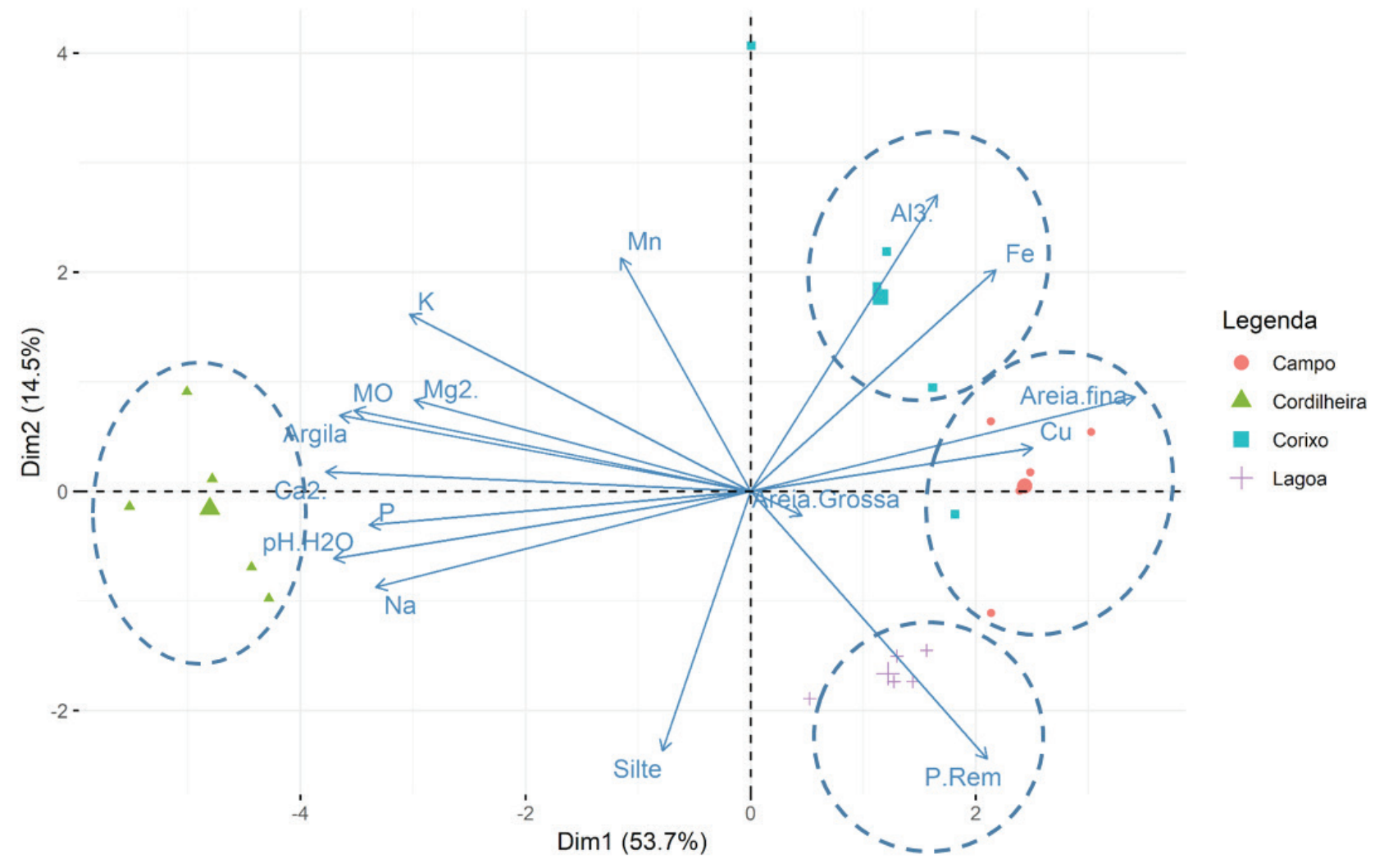

Figura 4. Análise de Componentes Principais (PCA) de atributos pedológicos superficiais $(0-20 \mathrm{~cm})$ dos Geoambientes do Pantanal do Abobral, estado do Mato Grosso do Sul, Brasil. MO - Matéria Orgânica; P-rem - Fósforo Remanescente.

Figure 4. Principal Components Analysis (PCA) of soil variables (depth 0-20 cm) at Pantanal do Abobral Geoenvironments, state of Mato Grosso do Sul, Brazil. MO - Organic Matter; P-rem - Remaining Phosphorus.

nutrientes do que os Geoambientes sazonalmente inundáveis. Ademais, há pouca acumulação de serapilheira devido à ação microbiana, propiciada pelo ambiente oxidante e estimulada pelos altos teores de $\mathrm{Ca}^{+2}$ (Cunha et al. 1985). Estas características explicam a maior fertilidade natural deste Geoambiente.

Nos Geoambientes sujeitos à inundação periódica (i.e., Lagoa Intermitente, Campo com Cambará e Corixo) ocorrem, de maneira generalizada, Planossolos (Queiroz et al. 2017). O principal processo pedogenético nestes Geoambientes é a podzolização, ou seja, translocação de argila de horizontes superficiais seguida de sua acumulação em horizontes subsuperficiais, resultando em um expressivo gradiente textural (Resende et al. 2002). Em razão disto, estes Geoambientes sujeitos a inundações anuais apresentaram os menores teores de argila em superfície (Tabela 2). Por conseguinte, a Cordilheira, ambiente não inundável e sem translocação de argila, possui os maiores teores de argila nos primeiros $20 \mathrm{~cm}$, seguida do Geoambiente Corixo, cujo horizonte textural inicia-se a $14 \mathrm{~cm}$ da superfície (Queiroz et al. 2017) (Tabela 2). Na planície de inundação, há maiores valores de areia grossa (AG) e fina (AF) (Tabela 2; Figura 2). Vale ressaltar que tais resultados não evidenciam Geoambientes arenosos, pois predominam Planossolos, mas devido ao gradiente textural há horizontes superficiais arenosos. A Lagoa Intermitente e Campo com Cambará, por terem um menor teor de argila, foram significativamente diferentes do Corixo em sua CTC (valores te T) (Tabela 2).

Devido à ocorrência de Planossolos, a drenagem vertical no perfil é muito lenta, ocasionando um lençol d'água suspenso temporariamente, e intensificando o regime de saturação hídrica a que a vegetação está sujeita (Couto et al. 2017, Queiroz et al. 2017).

O Campo com Cambará e Corixo apresentaram $\mathrm{pH}_{\text {н2O }}$ significativamente mais ácidos, situação que afeta a disponibilidade de nutrientes para a 
vegetação (Meurer 2007). Além disso, em pH ácido o alumínio se torna muito solúvel, o que justifica o maior valor de Índice de Saturação por Alumínio no Campo ( $m=35,3 \%)$, podendo se tornar tóxico para a vegetação.

Nas áreas com inundações mais prolongadas, como o caso da Lagoa Intermitente e Corixo, com formas embaciadas, os teores mais elevados de $\mathrm{H}^{+}$estão relacionados à decomposição lenta da matéria orgânica, que é depositada em razão da cheia, agindo como efeito tampão sobre o pH do solo (Abreu et al. 2007). Com efeito, a acidez potencial $(\mathrm{H}+\mathrm{Al})$ na Lagoa Intermitente é representada apenas por íons $\mathrm{H}^{+}$(Tabela 2).

Os Planossolos na planície de inundação possuem horizontes superficiais eluviados e ácidos, sujeitos ao ciclo de umedecimento e dessecação. Essa situação favorece o processo de ferrólise, em que a oxidação de ferro $\left(\mathrm{Fe}^{+2}\right)$ libera íons $\mathrm{H}^{+}$. Estes últimos são capazes de deslocar outros cátions básicos dos sítios de troca, resultando na destruição e intercalação de argilominerais, o que incrementa o contraste textural entre horizontes (Kampf \& Curi 2012).

Quando saturado com água, o pedoambiente torna-se redutor (i.e., com baixo potencial de oxirredução) e o Fe torna-se disponível, principalmente com o aumento da acidez, em que a velocidade de redução do elemento é maior (Sousa et al. 2009). Por isso houve um maior teor de Fe disponível nos Geoambientes da planície de inundação, do que na Cordilheira, ambiente este, oxidante e mais alcalino.

O Zn tende a ser altamente fixado pelas argilas (Kabata-Pendias \& Pendias 1992). Já o Mn tem maior mobilidade que o Fe (Sousa et al. 2009) e tende a migrar no perfil do solo conjuntamente ao processo de podzolização, o que justifica um maior valor de manganês na Cordilheira do que na Lagoa Intermitente e Campo com Cambará (Tabela 2).

Além de passar por inundações periódicas, a planície pantaneira possui o lençol freático elevado em boa parte do ano (Couto et al. 2017). Dessa maneira há de considerar grande influência da oscilação dolençol freático não só nos processos pedológicos, mas igualmente no estabelecimento das comunidades vegetais. Para maioria das plantas um ambiente saturado por água se torna restritivo, gerando um gradiente vegetacional em que elementos herbáceos ocupam áreas de pior drenagem e menor altitude, e elementos arbóreos os pontos mais altos e livres de inundação (Ferreira-Junior 2009).

Por outro lado, Cunha \& Junk (2009) comentam que diferentes comunidades vegetais podem ser encontradas num mesmo gradiente de inundação, o que evidencia que complexas interações entre fatores abióticos podem criar diferentes condições de habitat. Como demonstrado aqui, embora as unidades da paisagem estejam sujeitas a diferentes regimes de umidade, é possível estratificar diferentes coberturas vegetais a partir de atributos químicos do solo, especialmente no que tange a fertilidade natural do solo (Cardoso et al. 2016).

A presença numerosa de acuri (S. phalerata) na Cordilheira aponta a espécie como indicadora de solos básicos (Ratter et al. 1988) e férteis (Pott \& Pott 1994). Assim, a alta densidade de acuri nas cordilheiras do Abobral indica a presença de carbonatos no solo, e registra presença de árvores calcífilas nessa região (Cunha et al. 1985). Já a presença de B. balansae, é indicadora da condição de não alagamento (Cunha 1990), situação que permite originar um sub-bosque. No entanto, em recente levantamento florístico na região do Abobral, Andrade (2017) aponta grandes efeitos de degradação antrópica, como a intensa propagação da $B$. balansae, que tem rápido crescimento em clareiras e áreas desmatadas.

Para Cunha et al. (1985), o elenco florístico encontrado nas Cordilheiras do Abobral não é de Cerrado, como classificou o projeto RADAMBRASIL (1982), e sim de florestas decíduas calcárias ou semidecíduas, também similares às cordilheiras do Pantanal de Barão de Melgaço, estado do Mato Grosso (Ferreira-Junior 2009). Essas vegetações são estritamente associadas a solos eutróficos e bem drenados (Arruda et al. 2013).

Já o Corixo, devido à sua condição de dreno na paisagem, está sujeito a um maior arraste de partículas. $\mathrm{O}$ acúmulo de serapilheira é propiciado pela vegetação arbórea e pela lenta mineralização da matéria orgânica, causada pelo ambiente mais redutor, outro fator que contrasta a dinâmica deste ambiente com as Cordilheiras.

Conforme a paisagem transiciona para a planície de inundação há substituição da fitofisionomia 
florestal por campos, os quais dominam os pontos de menor altitude. Cunha (1980) explica que nas áreas aplainadas pantaneiras, em geral, a vegetação é de baixo porte e rala, como foi registrado na Lagoa Intermitente, não havendo adensamento da vegetação devido à seleção pelo regime hídrico, intensificado pelo horizonte subsuperficial pouco permeável. Algumas espécies de plantas adaptadas a solos inundados conseguem capturar $\mathrm{O}_{2}$ atmosférico através das folhas e transportá-lo para as raízes via aerênquima ou espaços intracelulares. Isto permite às raízes oxidarem a rizosfera $\mathrm{e}$ colonizarem solos e sedimentos fortemente reduzidos (Sousa et al. 2009). No entanto conforme Junk et al. (1989) expõem, nos ambientes inundáveis as espécies anuais e temporárias, como as macrófitas aquáticas, mantém um estágio inicial de sucessão, resultado da constante renovação vegetal pelo pulso de inundação.

No Campo com Cambará, Geoambiente com solo de menor fertilidade natural, destacase $V$. divergens, que se encontra em estágio de sucessão inicial nesse ambiente. É uma espécie arbórea de origem Amazônica (Pott \& Pott 1994) e considerada invasora natural das áreas campestres do Pantanal (Pott \& Pott 1994, Cunha \& Junk 2004). Esta suporta elevado nível de saturação hídrica, seja como plântula ou indivíduo adulto, com rápido crescimento sob alta luminosidade e de ampla ocorrência no gradiente de inundação (Rebelatto 2010), além de tolerante e acumuladora de alumínio (Haridasan 2000, Arieira \& Cunha 2012). Embora o valor de Al disponível no Campo com Cambará não tenha se diferido dos demais Geoambientes, a menor soma de bases (valor SB) deste ambiente resultou em alta saturação por Al nos sítios de troca (valor m) (Tabela 2). Esse fato também é registrado em formação de Cambarazal em planície de inundação no Pantanal de Barão de Melgaço, estado do Mato Grosso, sobre Plintossolo Alumínico abrúptico, e de baixa fertilidade natural (Ferreira-Junior 2009).

Dessa maneira, ao ter um pedoambiente ácido e com alta saturação em alumínio em superfície $(\mathrm{m}=35,3 \%)$, o Campo com Cambará reproduz um ambiente favorável à ocupação por Cambará e à formação monodominante. Além da tendência aluminícola, em anos mais chuvosos árvores como $V$. divergens e arbustos tolerantes à inundação tendem a colonizar áreas por extravasamento fluvial (Arieira \& Cunha 2012).

As formações monodominantes cobrem grandes áreas no Pantanal. Nos arredores do Pantanal do Abobral há registros de carandazal (Copernicia alba; Arecales, Arecaceae), e nos Pantanais do Miranda e Nabileque de paratudal (Tabebuia aurea; Lamiales, Bignoniaceae), e que já foram associados a água e sedimentos alcalinos (Bueno et al. 2014). Andrade (2017) registrou recentemente relatos de moradores tradicionais da área do Abobral de um crescente aumento de formações monodominantes, principalmente cambarazal e canjiqueiral (Byrsonima cydoniifolia; Malpighiales, Malpighiaceae).

Arieira \& Cunha (2012) consideram que o Cambará tenha preferência por solos argilosos, no entanto este indício ainda carece de pesquisas, uma vez que Ferreira-Junior (2009) registrou Cambarazal sobre solos alumínicos e arenosos e, no estudo de Queiroz et al. (2017), o Geoambiente Campo com Cambará apresentou menores valores de argila em todo seu perfil, com textura mais arenosa.

Assim, o presente estudo demonstrou que as descontinuidades da paisagem e nas formações vegetais são estritamente associadas às características físico-químicas dos solos, provando ser um dos principais estratificadores do ambiente, ao lado do nível do lençol freático, sempre próximo à superfície no caso do Pantanal.

A Cordilheira, unidade da paisagem livre de inundação, há presença de solo naturalmente fértil e básico, que suporta o estabelecimento de mata semidecídua com sub-bosque. Na planície de inundação, os Geoambientes tornam-se mais ácidos, com menores participações das bases e maior participação de metais (micronutrientes) nos sítios de troca. No Geoambiente Corixo a vegetação funciona como mata ciliar sobre solo com características redutoras, resultando em acúmulo de serapilheira e lenta mineralização orgânica. $\mathrm{Na}$ Lagoa Intermitente a constante saturação hídrica favorece vegetação tolerante à inundação e macrófitas aquáticas; enquanto o Campo com Cambará apresentou a menor fertilidade natural, e condições propícias a uma maior saturação por alumínio, sendo recoberto por gramíneas e processo inicial de colonização por $V$. divergens.

Os diferentes Geoambientes que compõem a paisagem do Pantanal do Abobral se mostraram 
distintos pedologicamente, com o quadro vegetacional respondendo diretamente às características edáficas superficiais.

\section{AGRADECIMENTOS}

Os autores agradecem à Fundação de Apoio ao Desenvolvimento do Ensino, Ciência e Tecnologia do Estado de Mato Grosso do Sul (FUNDECT) e ao Instituto Nacional de Pesquisa do Pantanal (INPP), pelo financiamento e apoio que permitiram a realização desta pesquisa; e ao pesquisador Daniel Arruda pelas valiosas contribuições ao trabalho.

\section{REFERÊNCIAS}

Abreu, E. M. A., Fernandes, A. R., \& Ruivo, M. L. P. 2007. Variação temporal e vertical de atributos químicos de um gleissolo do rio Guamá cultivado com Canaranas. Revista Brasileira de Ciência do Solo, 31(2), 277-285. DOI: 10.1590/ S0100-06832007000200010

Ab'Saber, A. N. 1988. O Pantanal Mato-Grossense e a Teoria dos Refúgios. Revista Brasileira de Geografia, 50(2), 9-57.

Alho, C. J. R. 2005. The Pantanal. In: L. H. Fraser, \& P. A. Keddy (Eds.), The World's largest wetlands: Ecology and conservation. pp. 203-271. Cambridge: Cambridge University Press.

Andrade, B. S. 2017. Análise da paisagem de ambientes florestais não inundáveis no Pantanal do Abobral, Mato Grosso do Sul. Dissertação de mestrado. Programa de Meio Ambiente e Desenvolvimento Regional da Universidade Anhanguera. p. 91.

Arieira, J., \& Cunha, C. N. 2012. Estrutura populacional do cambará (Vochysia divergens Pohl, Vochysiaceae), espécie monodominante em floresta inundável no Pantanal MatoGrossense. Oecologia Australis, 16(4), 819-831. DOI: 10.4257/oeco.2012.1604.07

Arruda, D. M., Ferreira-Junior, W. G., Duque-Brasil, R., \& Schaefer, C. E. R. 2013. Phytogeographical patterns of dry forests sensu stricto in northern Minas Gerais State, Brazil. Anais da Academia Brasileira de Ciências, 85(2), 623-634. DOI: 10.1590/s0001-37652013000200011

Assine, M. L. 2003. Sedimentação na Bacia do Pantanal mato-grossense, Centro-Oeste do Brasil. Tese de doutorado. Departamento de
Geologia Sedimentar da Universidade Estadual Paulista. p. 115.

Bueno, M. L., Damasceno-Junior, G. A., Pott, A., Pontara, V., Seleme, E. P., Fava, W. S., Salomão, A. K. D., \& Ratter, J. A. 2014. Structure of arboreal and herbaceous strata in a neotropical seasonally flooded monodominant savanna of Tabebuia aurea. Brazilian Journal of Biology, 74(2), 325-337. DOI: 10.1590/1519-6984.16612

Cardoso, E. L., Santos, S. A., Urbanetz, C., Carvalho Filho, A., Naime, U. J., Silva, M. L. N., \& Curi, N. 2016. Relação entre solos e unidades da paisagem no ecossistema Pantanal. Pesquisa Agropecuária Brasileira, 51(9), 1231-1240. DOI: 10.1590/S0100-204X2016000900023

Costa, C. P. 2013. Padrões da distribuição de plantas arbóreo-arbustivas em meso-escala no Pantanal de Mato Grosso. Tese de doutorado. Departamento de Engenharia Florestal da Universidade de Brasília. p.117.

Couto, E. G., Oliveira, V. A., Beirigo, R. M., Oliveira Junior, J. C., Nascimento, A. F., \& Vidal-Torrado, P. 2017. Solos do Pantanal Mato-Grossense. In: N. Curi, J. C. Ker, R. F. Novais, P. Vidal-Torrado, \& C. E. G. R. Schaefer (Eds.), Pedologia. Solos dos Biomas Brasileiros. pp. 303--352. Viçosa: Sociedade Brasileira de Ciência do Solo.

Cunha, C. N., \& Junk, W. J. 2004. Year-to-year changes in water level drive the invasion of Vochysia divergens in Pantanal grasslands. Applied Vegetation Science, 7(1), 103-110. DOI: 10.1111/j.1654-109x.2004.tb00600.x

Cunha, C. N., \& Junk, W. J. 2009. A preliminary classification of habitats of the Pantanal of Mato Grosso and Mato Grosso do Sul, and its relation to national and international wetland classification systems. In: W. J. Junk, C. J. Silva, C. N. Cunha, \& K. M. Wantzen (Eds.), The Pantanal: Ecology, biodiversity and sustainable management of a large neotropical seasonal wetland. pp. 127-141. Moscow: Pensoft Publishers.

Cunha, C. N. 1990. Estudos florísticos e fitofisionômicos das principais formações arbóreas do Pantanal de Poconé - MT. Dissertação de mestrado. Universidade Estadual de Campinas, Campinas. p. 146.

Cunha, N. G. 1980. Considerações sobre os solos da sub-região da Nhecolândia, Pantanal matogrossense. Circular Técnica 01. Corumbá: Empresa Brasileira de Pesquisa Agropecuária/ 
Centro de Pesquisa Agropecuária do Pantanal: p. 45 .

Cunha, N. G. 1985. Dinâmica de nutrientes em solos arenosos no Pantanal Mato-Grossense. Circular Técnica 17. Corumbá: Empresa Brasileira de Pesquisa Agropecuária/Centro de Pesquisa Agropecuária do Pantanal: p. 70.

Cunha, N. G., Pott, A., \& Gonçalves, A. R. 1985. Solos calcimórficos da sub-região do Abobral, Pantanal Mato-grossense. Circular Técnica 19. Corumbá: Empresa Brasileira de Pesquisa Agropecuária/Centro de Pesquisa Agropecuária do Pantanal: p. 52.

EMBRAPA - Empresa Brasileira de Pesquisa Agropecuária. 2017. Manual de métodos de análise de solos. Brasília, DF: Embrapa: p. 574.

Ferreira-Júnior, W. G. 2009. Análise de gradientes vegetacionais e pedogeomorfológicos em floresta, cerrado e campo no Pantanal matogrossense, Barão de Melgaço, Mato Grosso. Tese de doutorado. Departamento de Biologia Vegetal da Universidade Federal de Viçosa. p. 190.

Haridasan, M. 2000. Nutrição mineral de plantas nativas do cerrado. Revista Brasileira de Fisiologia Vegetal, 12(1), 54-64.

Jobbágy,E.G., \&Jackson, R. B. 2001.The distribution of soil nutrients with depth global patterns and the imprint of plants. Biogeochemistry, 53(1), 51-77.

Junk, W. J., Bayley, P. B., \& Sparks, R. S. 1989. The flood pulse concept in river-floodplain systems. Canadian Special Publication of Fisheries and Aquatic Sciences, 106(1), 110-127.

Junk, W. J., Cunha, C. N., Wantzen, K. M., Petermann, P., Strüssmann, C., Marques, M. I., \& Adis, J. 2006. Biodiversity and its conservation in the Pantanal of Mato Grosso, Brazil. Aquatic Sciences, 68(3), 278-309.

Kabata-Pendias, A., \& Pendias, H. 1992. Trace elements in soils and plants. 2a ed. Florida: CRC Press. p. 363.

Kampf, N., \& Curi, N. 2012. Formação e evolução do solo (Pedogenêse). In: J. C. Ker, N. Curi, C. E. G. R. Schaefer, \& P. Vidal-Torrado (Eds.), Pedologia - Fundamentos. pp. 207-302. Viçosa: Sociedade Brasileira de Ciência do Solo.

Meurer, E. J. 2007. Fatores que influenciam o crescimento e desenvolvimento das plantas. In: R. F. Novaes, V. H. Alvarez, N. F. Barros, R.
L. F. Fontes, R. B. Cantarutti, \& J. C. L. Neves (Eds.), Fertilidade do solo. pp. 65-90. Viçosa: Sociedade Brasileira de Ciência do Solo.

Novais, R. F., Smyth, T. J., \& Nunes, F. N. 2007. Fósforo. In: R. F. Novaes, V. H. Alvarez, N. F. Barros, R. L. F. Fontes, R. B. Cantarutti, \& J. C. L. Neves (Eds.), Fertilidade do solo. pp. 471-550. Viçosa: Sociedade Brasileira de Ciência do Solo.

Por, F. D. 1995. The Pantanal of Mato Grosso (Brazil) - World's Largest Wetlands. Dordrecht, Netherlands: Kluvwer Academic Publishers: p. 122.

Pott, A., \& Pott, V. J. 1994. Plantas do Pantanal. Corumbá: Empresa Brasileira de Pesquisa Agropecuária: p.320.

Queiroz, R. F. P., Corrêa, G. R., Gradella, F. S., Bernardes, G. M. P., Schaefer, C. E. G. R., \& Ruivo, M. L. P. 2017. Geoambientes no Pantanal do Abobral, Mato Grosso do Sul, Brasil. Boletim do Museu Paraense Emílio Goeldi - Ciências Naturais. 12(2), 277-291.

RADAMBRASIL - Ministério de Minas e Energia. Secretaria Geral. 1982. Folha SE. 21 Corumbá e parte da Folha SE. 20, Geologia, Pedologia, Geomorfologia, Vegetação e Uso e Ocupação da Terra. Vol. 27, Rio de Janeiro.

Ratter, J. A., Pott, A., Cunha, C. N., \& Haridasan, M. 1988. Observations on wood vegetation types in the Pantanal and at Corumbá, Brazil. Notes from the Royal Botanic Garden, 45(3), 503-525.

Ravaglia, A. G., Santos, S. A., Pellegrin, L. A., Rodela, L. G., \& Barbosa, A. J. 2010. Classificação preliminar das paisagens da sub-região do Abobral, Pantanal, usando imagens de satélite. Comunicado Técnico 82. Corumbá: Embrapa Pantanal: p. 4.

Rebelatto, L. 2010. Padrões sazonais e espaciais de distribuição e diversidade de herbáceas no Pantanal de Poconé - MT. Tese de doutorado. Instituto de Ciências Biológicas da Universidade Federal de Minas Gerais. p. 162.

Resende, M., Curi, N., Rezende, S. B., \& Corrêa, G. F. 2002. Pedologia: Base para distinção de ambientes. Viçosa: Editora UFV: p. 338.

Schaefer, C. E. G. R., Mendonça, B. A. F., FerreiraJunior, W. G.F., Valente, E. L., \&Corrêa, G. R. 2012. Relações solo-vegetação em alguns ambientes brasileiros: fatores edáficos e florística. In: S. V. Martins (Ed.), Ecologia de florestas tropicais do Brasil. pp. 252-293. Viçosa: Editora UFV. 
Sepúlveda, J. J. O. 2016. Conservação, grau de ameaça e monitoramento participativo da biodiversidade por meio do turismo da subregião do Abobral no Sul do Pantanal brasileiro. Dissertação de mestrado. Universidade para o Desenvolvimento do Estado e da Região do Pantanal. p. 90.

Silva, J. S. V., \& Abdon, M. M. 1998. Delimitação do Pantanal Brasileiro e suas Sub-regiões. Pesquisa Agropecuária Brasileira, 33, 1703-1711.

Sousa, R. O., Vahl, L. C., \& Otero, X. L. 2009. Química dos solos alagados. In: V. F. Melo, \& L. R. F. Alleoni (Eds.), Química e mineralogia do solo, Parte II. pp. 485-528, Viçosa: Sociedade Brasileira de Ciência do Solo.

Submetido em: 11/09/2018 Aceito em: 22/06/2019

Publicado online: 16/12/2019

Editores Associados: Camila Aoki, Gudryan J.

Barônio \& Arnildo Pott 